\title{
Megakaryopoiesis and Myelofibrosis in Chronic Myeloid Leukemia after Allogeneic Bone Marrow Transplantation: An Immunohistochemical Study of 127 Patients
}

Juergen Thiele, M.D., Hans Michael Kvasnicka, M.D., Dietrich Wilhelm Beelen, M.D., Uta Flucke, M.D., Christian Spoer, Svetlana Paperno, Lutz-Dietrich Leder, M.D., Ulrich Wilhelm Schaefer, M.D. Institutes of Pathology, Universities of Cologne (JT, HMK, UF, ChS, SP), Cologne, Germany, and Essen (LDL), Essen, Germany; and Department of Bone Marrow Transplantation (DWB, UWSch), University of Essen, Essen, Germany

An immunohistochemical and morphometric study was performed on 363 trephine biopsies of the bone marrow derived from 127 patients with chronic myeloid leukemia at standardized end points before and after allogeneic bone marrow transplantation (BMT). The purpose of this investigation was to evaluate features of $\mathrm{CD}^{+} 1^{+}$megakaryopoiesis related to successful engraftment. Further, we tried to elucidate possible associations of this lineage, including precursor cells, with the platelet count and reticulin fibrosis during the pretransplant and, specifically, post-transplant periods. A significant correlation was recognizable between the quantity of $\mathrm{CD}^{+} 1^{+}$megakaryocytes and the platelet values before BMT and also after completed hematopoietic recovery. In the very early post-transplant period, which is associated with severe thrombocytopenia, patchy regeneration of disarranged hematopoiesis occurred, including dysplastic megakaryocytes. According to planimetric measurements after BMT, the atypical micromegakaryocytes characteristic for chronic myeloid leukemia disappeared, and the engrafted donor bone marrow revealed a prevalence of normal-size cells of this lineage. On the other hand, normalization of megakaryocyte size was absent in sequential examinations of the few patients with a leukemic relapse who had a predominance of atypical dwarf forms comparable with chronic myeloid leukemia. Before BMT occurred, reticulin fiber density was significantly correlated with the num-

Copyright (C) 2000 by The United States and Canadian Academy of Pathology, Inc.

VOL. 12, NO. 1, P. 129, 2000 Printed in the U.S.A.

Date of acceptance: August 2, 2000.

Funding was sponsored by the Dr. M. Scheel Foundation for Cancer Research (contract grant 70-2134-Th1).

Address reprint requests to: Juergen Thiele, M.D., Institute of Pathology, University of Cologne, Joseph-Stelzmannstrasse 9, D-50924 Cologne, Germany; fax: +49-0221-4786360. ber of $\mathrm{CD}^{+} 1^{+}$megakaryocytes and its precursor cell population. In 34 patients with myelofibrosis that occurred after myelo-ablative therapy and BMT, an initial regression was followed by an insidious recurrence of fibers concentrated in the areas of regenerating hematopoiesis. This postgraft reappearance of reticulin fibrosis was significantly associated with the quantity of megakaryocytes. Regarding engraftment parameters, pretransplant presence of (reticulin) myelofibrosis exerted a distinctive impact because of a delayed hematopoietic reconstitution according to standard clinical criteria. In line with this finding, slowed engraftment was also significantly related with higher pretransplant megakaryocyte and platelet counts.

KEY WORDS: Bone marrow biopsy, Bone marrow transplant, Chronic myeloid leukemia, Megakaryocyte precursor, Megakaryopoiesis, Myelofibrosis.

Mod Pathol 2000;12(1):129-138

After allogeneic bone marrow transplantation (BMT) as the only therapeutic-curative option for patients with chronic myeloid leukemia (CML), hematopoietic reconstitution is generally defined by the achievement of certain parameters. In addition to a self-sustaining absolute neutrophil count exceeding $0.5 \times 10^{9} / \mathrm{L}$, normal graft function is assumed with maintenance of an unsupported thrombocyte count greater than $20.0 \times 10^{9} / \mathrm{L}$ until 30 days after BMT (1-7). Regarding this last parameter, to date, no information is available about quantitative and morphologic features of megakaryopoiesis during the peri- and post-transplant periods. Because CML is characterized by the occurrence of a distinctive population of atypical dwarf-like megakaryocytes or micromegakaryocytes (8-12), it might be speculated that successful engraftment is consistent 
with a reappearance of normal-size megakaryocytes. The current hypothesis for the pathogenesis of myelofibrosis involves the intramedullary release of certain growth factors from defective and abnormal megakaryocytes (13-15), in particular involving precursors of this cell lineage $(16,17)$. In the context of this complex functional association, the question arises whether the amount of reticulin fibers is correlated with the number of mature and immature megakaryocytes (promegakaryoblasts-megakaryoblasts) as well.

The present study histochemically and morphometrically investigated 363 sequential bone marrow trephine biopsies derived from 127 patients before and after allogeneic BMT for CML and was focused on correlations between number of $\mathrm{CD}^{+} 1^{+}$ megakaryocytes and platelet count; features of megakaryo- and thrombocytes accompanying successful or delayed engraftment and leukemic relapse; and association of megakaryopoiesis and their precursors with fiber density during the pretransplant and, specifically, post-transplant periods of hematopoietic recovery.

\section{PATIENTS AND METHODS}

\section{Patients}

We retrospectively evaluated 127 patients (75 men, 52 women; median age at time of BMT, $35 \mathrm{y}$ ) presenting with the first chronic (stable) phase of CML and allogeneic BMT. Patients received marrow grafts from human leukocyte antigen-compatible family members or from unrelated donors at a single referral transplantation center; standard procedures were followed. Details regarding history, clinical findings, and treatment characteristics have been reported in detail elsewhere (2). Homogeneity of the cohort we studied concerning specifications of relevant transplant-associated data was established by including about $80 \%$ sibling donors, with more than $70 \%$ having an identical human leukocyte antigen constellation and the same conditioning regimen (pretransplant myelo-ablative treatment) in $90 \%$ of patients. The end point of hematologic reconstitution with normal graft function was assumed if patients achieved transplantderived absolute neutrophil counts greater than 0.5 $\times 10^{9} / \mathrm{L}$ and self-sustaining thrombocyte counts exceeding $20.0 \times 10^{9} / \mathrm{L}$ until 30 days after BMT. Delayed engraftment was diagnosed if these thresholds were not present at Day 30, and graft failure
TABLE 1. Time and End Points Regarding the Different Bone Marrow Biopsies in Our patients Before and After Transplantation (BMT) Procedures

\begin{tabular}{clcc}
\hline End points & \multicolumn{1}{c}{ Period } & $\begin{array}{c}\text { Number of } \\
\text { Patients }\end{array}$ & $\begin{array}{c}\text { Mean } \pm \text { SD } \\
\text { (Days) }\end{array}$ \\
\hline 0 & Pretreatment biopsy & 33 & - \\
1 & Before BMT to Day 0 (BMT) & 127 & $24 \pm 23$ \\
2 & After BMT (Day 0) to Day 30 & 25 & $22 \pm 6$ \\
3 & Day 31 to Day 45 & 52 & $38 \pm 4$ \\
4 & Day 46 to Day 119 & 55 & $64 \pm 17$ \\
5 & Day 120 to Day 365 & 18 & $179 \pm 61$ \\
\hline
\end{tabular}

A patient may have up to six examinations at significantly different intervals.

BMT, bone marrow transplantation.

was declared if cell counts did not reach these levels at standardized end points (Table 1). After BMT, a successful engraftment according to the abovementioned standard criteria (1-7) was established in 95 patients, a delayed hematopoietic reconstitution in 32 , primary failure to engraft in 4 , and a leukemic relapse in 19 during a post-transplant observation time ranging from 1 to 10 years.

\section{Bone Marrow Biopsies}

Bone marrow trephine biopsy samples were taken from the posterior iliac crest at significantly different intervals before and after BMT. For this reason, standardized end points have been chosen so that we may properly assess all the parameters under study. These standardized end points are outlined in detail in Table 1. Fixation of samples was carried out in a lowconcentrate phosphate-buffered aldehyde solution (2 to $3 \%$ ) for 12 to 48 hours. Further processing included decalcifying the samples for 3 to 4 days in $10 \%$ buffered ethylenediamine tetra-acetic acid, $\mathrm{pH}$ 7.2, embedding them in paraffin, and using several routine staining techniques, which involved Giemsa staining, periodic acid-Schiff (periodic acid-Schiff reagent), naphthol-AS-D-chloroacetate esterase, Perls' reaction for iron, and a silver impregnation method (Gomori's technique). For specific staining of megakaryopoiesis, the monoclonal antibody $\mathrm{Y}_{2} / 51$ directed against $\mathrm{CD} 61$ (anti-platelet glycoprotein IIIa) was applied (18). As has been previously recorded, identification of mature megakaryocytes and precursor cells of this lineage (promegakaryoblasts and megakaryoblasts) was achieved not only by regarding their different staining reactions with periodic acid-Schiff (mature megakaryocytes) and CD61 (total megakaryopoiesis), but also by their morphology (19).The monoclonal

FIGURE 1. (a-e) Megakaryocytes before (a) and after (b-e) bone marrow transplantation (BMT) in chronic myelogenous leukemia (CML). (a) Pretransplant bone marrow with micromegakaryocytes characterizing CML. (b) Bone marrow at Day 15 with slowed regeneration of erythro- and megakaryopoiesis reveals small-size megakaryocytes, some with dysplastic features surrounded by erythroid precursors and an interstitial edema. (c) At a later date (Day 25), other patients show large-size megakaryocytes with clumsy nuclear lobulation assembled in clusters in an edematous stroma. (d) Completed, transfusion-independent engraftment with grossly normal appearing bone marrow including megakaryocytes at Day 38. (e) Leukemic relapse in one patient (see Fig. 3) about 3 years after BMT reveals recurrence of atypical micromegakaryocytes (370×). 

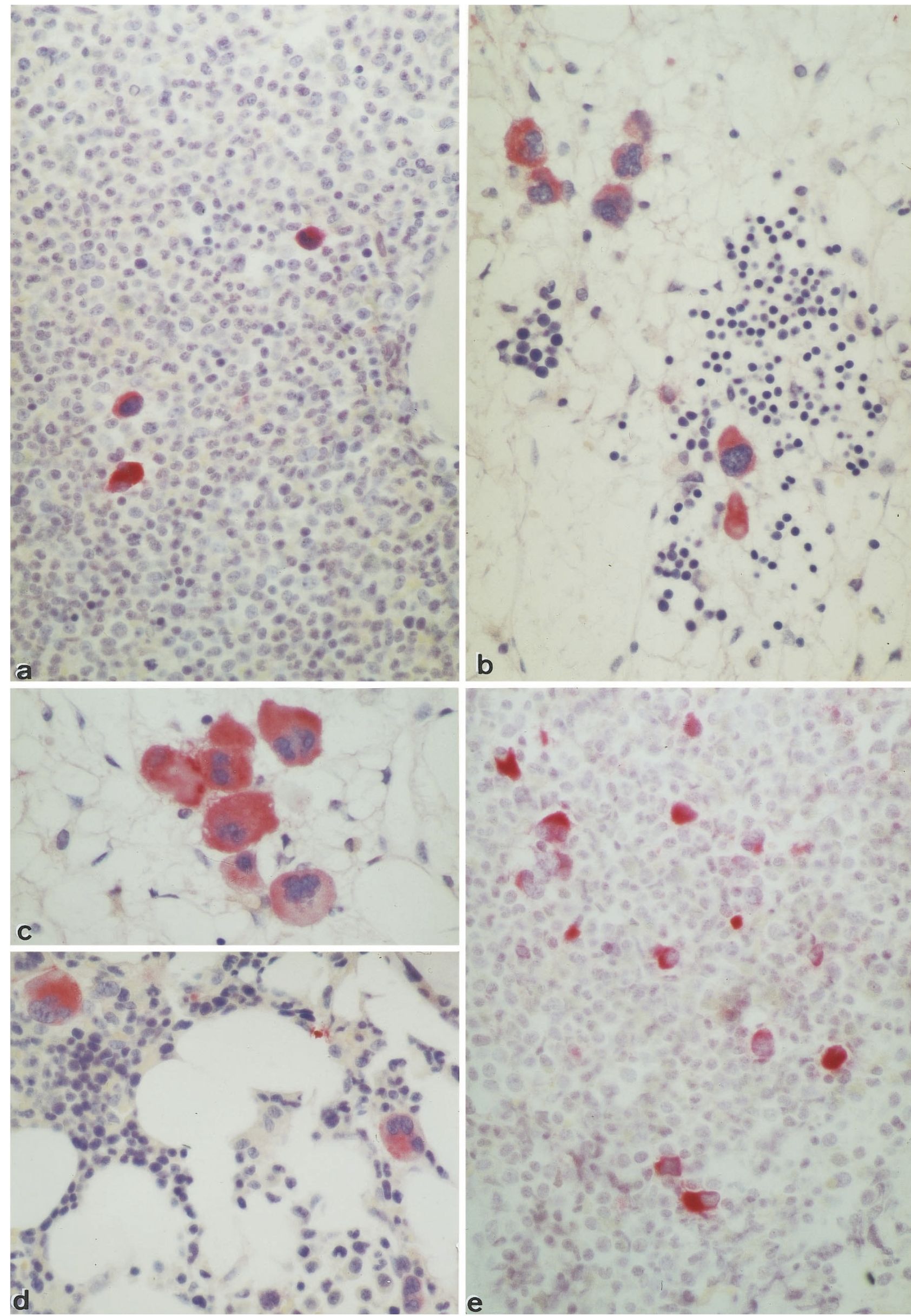


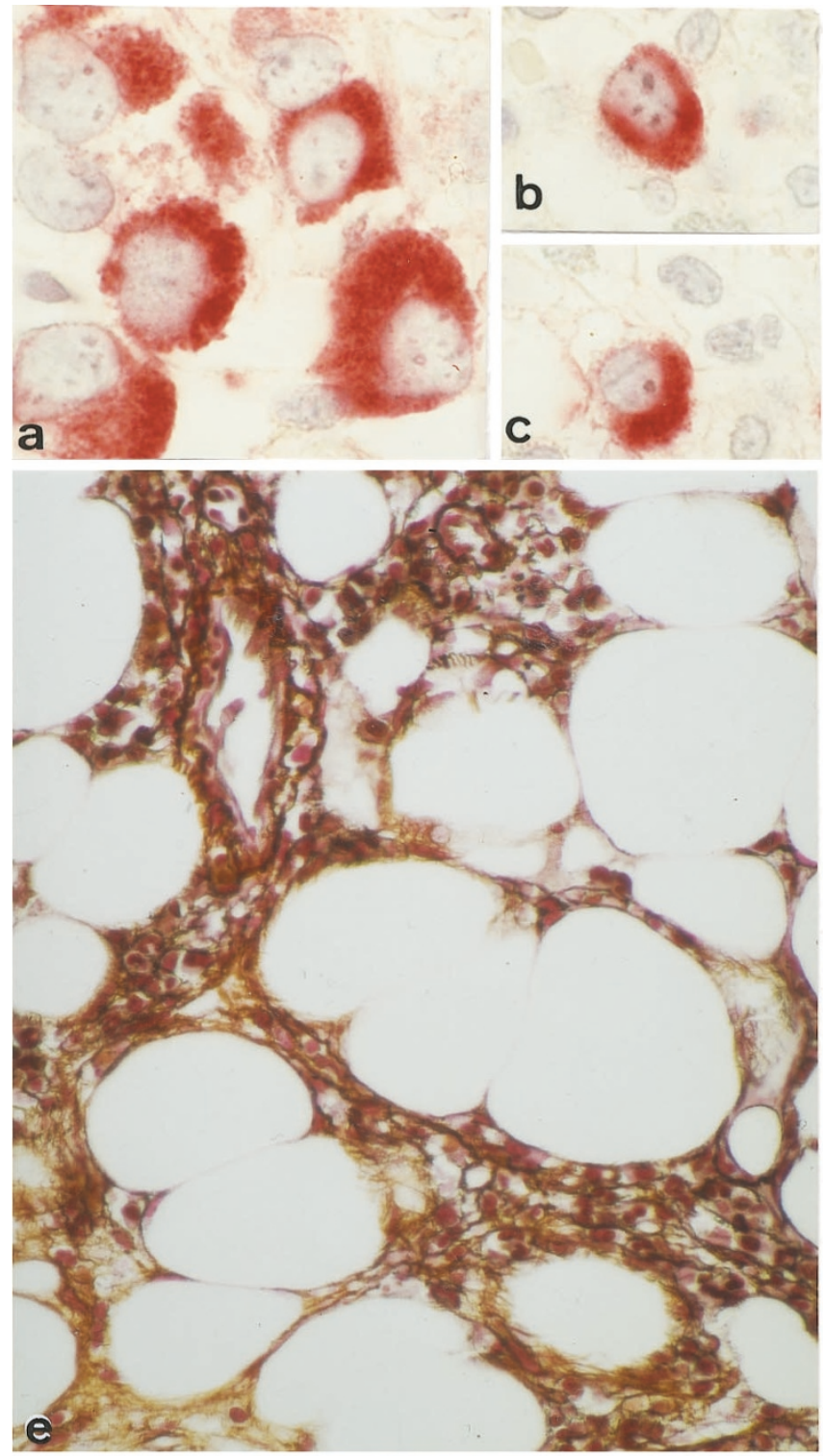

(17)

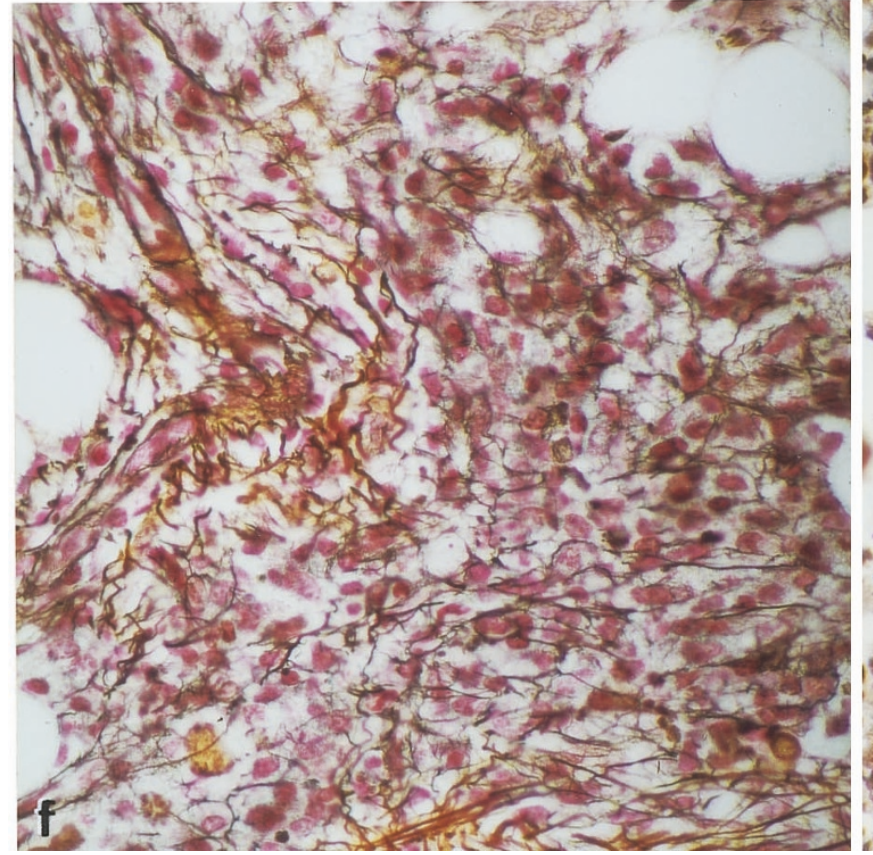

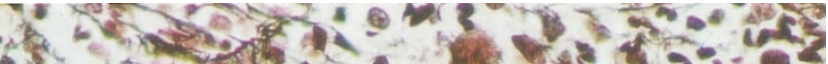

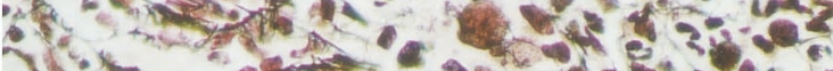

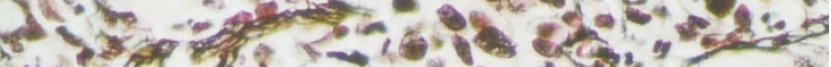

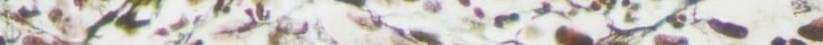

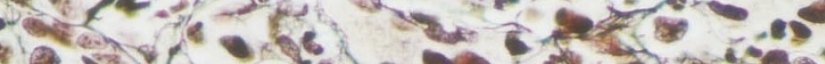

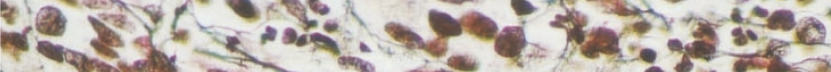

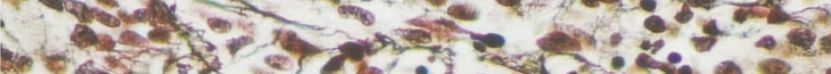

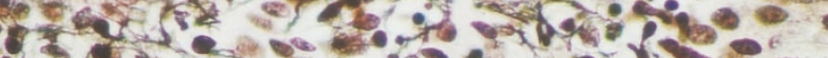

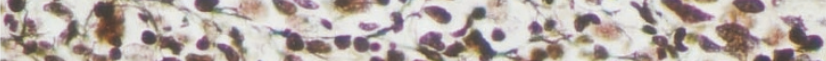

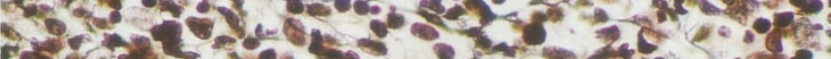

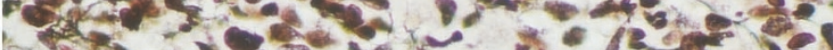

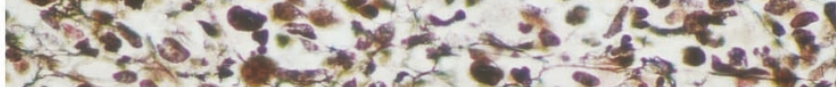

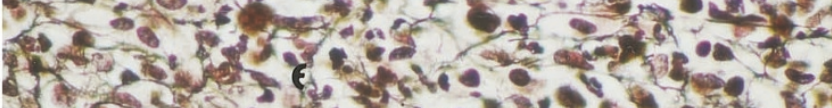

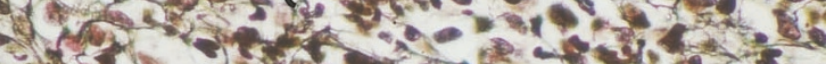

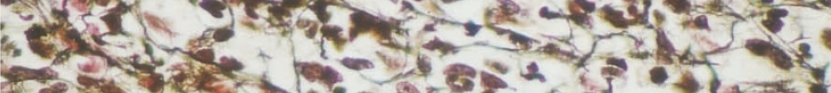

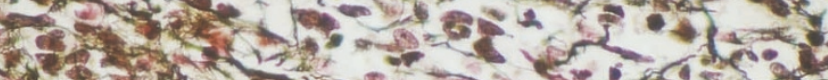

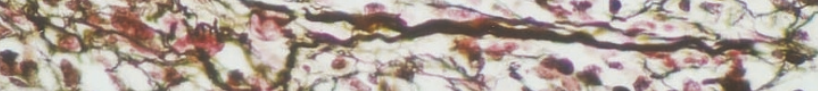

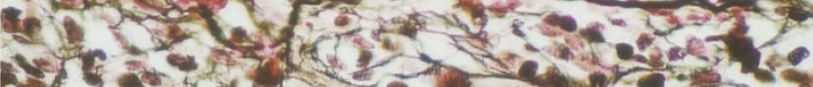

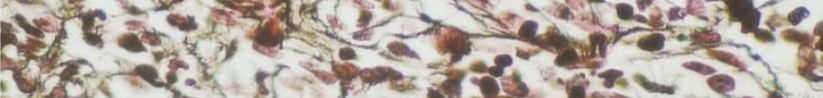

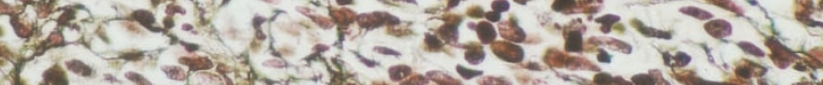

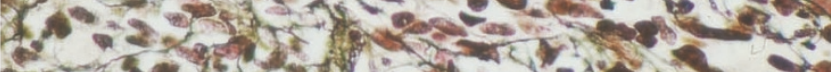

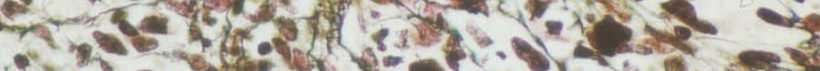

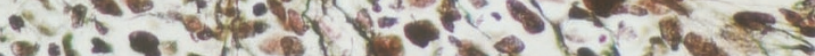

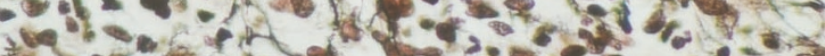

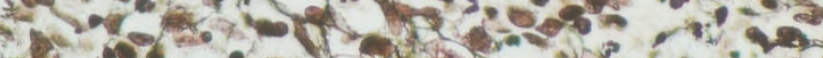

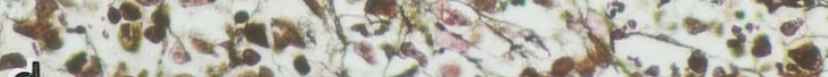

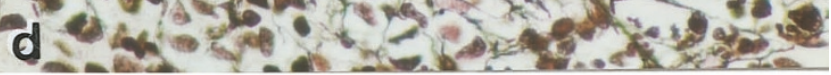

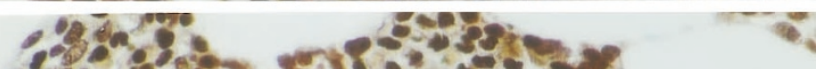
-

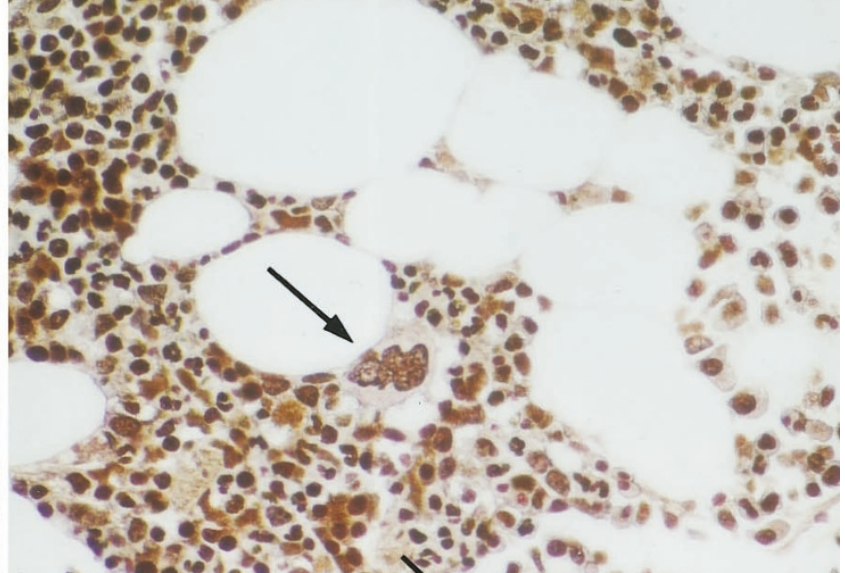

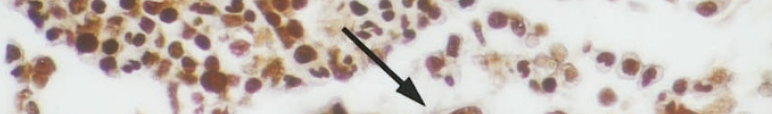

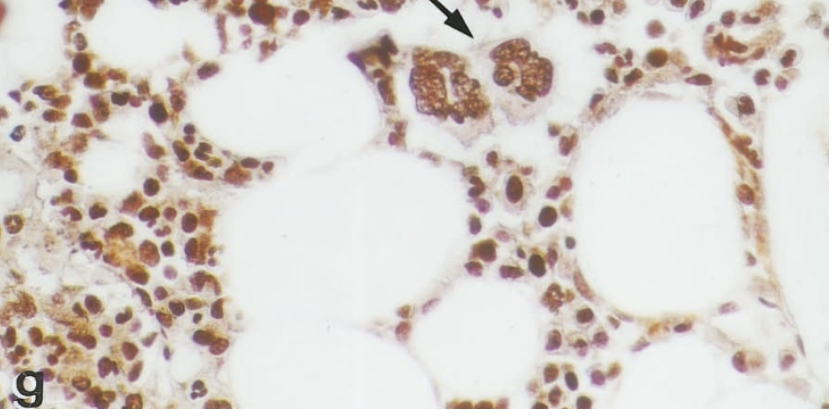


antibody $\mathrm{Y}_{2} / 51$ (CD61) and other reagents were purchased from Dako-Diagnostica GmbH (Hamburg, Germany). Details of staining procedures (APAAP method) have been previously reported $(10,19)$.

\section{Morphometry}

After periodic acid-Schiff reaction, silver impregnation, and immunostaining with CD61, morphometric analysis was performed by two manual optic planimeters (VIDAS-Zeiss-Kontron) and a specially developed program set (Optimas software) on large trephine biopsies with an artifact-free mean marrow area of $15.4 \pm 4.6 \mathrm{~mm}^{2}$. Frequencies of periodic acid-Schiff- and CD61-positive megakaryocytes $\left(\mathrm{CD}_{61}{ }^{+}\right)$, including atypical microforms and immature elements (pro- and megakaryoblasts), were calculated per square millimeter of marrow area by regarding the total biopsy specimen. Planimetric measurements of megakaryocyte size and allied parameters was carried out on CD61-stained biopsies and involved 6278 megakaryocytes in a randomly selected number of 25 patients with multiple representative examinations before and after BMT. After silver impregnation, density of argyrophilic (reticulin-collagen) fibers was determined by the point-intersection method by use of an ocular grid. Thus, the amount of fibers was expressed as intersections per area (expressed in square millimeters) of hematopoietic tissue (cellularity). Reference to cellularity was necessary to avoid the erroneous impression of a reduction in megakaryocyte numbers and fiber density after an expansion of adipose tissue or interstitial edema. This feature was particularly prominent after marrow-ablative therapy and during the post-transplant period in patients who experienced delayed hematopoietic recovery. For assessment of the transplant-specific changes in megakaryopoiesis, data on an age-matched control group of 25 patients (11 men and 14 women) were also included.

\section{Statistics}

Statistical analysis included calculation of correlation coefficients between hematologic and morphometric variables and Student's $t$ test as well as the nonparametric Mann-Whitney $U$ test to compare differences in mean values at pretransplant and post-transplant end points.

\section{RESULTS}

At diagnosis, the CML bone marrow was not only characterized by a predominance of granulocytic proliferation but also by a striking variability in the growth of atypical micromegakaryocytes (Figs. 1a, 2a). A significant correlation between the number of $\mathrm{CD} 1^{+}$megakaryocytes and the platelet count was recognizable in the trephine biopsy samples taken before BMT (Fig. 3). Compared with their pretransplant quantity after myelo-ablative therapy and BMT, frequency of this lineage, including precursor cells, decreased significantly (Table 2). This reduction was demonstrable in the first biopsies performed at Day 9 after BMT and was associated with severe thrombocytopenia that required appropriate supportive treatment. Contrasting normal bone marrow features trephine biopsies taken at this very early post-transplant period revealed not only a decrease in cellularity, but also a significant disarrangement of regenerating hematopoiesis occurring within patchy areas. Especially in patients with delayed engraftment, a conspicuous so-called scleredema of the interstitial space could be recognized (Fig. 1b, 1c), as well as megakaryocytes revealing a remarkable dysplastic aspect (Fig. 1b).

Cytologic abnormalities of megakaryocytes shortly after BMT were reflected by a corresponding shift of the nuclear-cytoplasmic ratio in comparison to later end points of examination (Table 3 ). Moreover, this stage presented with a significantly lowered platelet number (Table 2), probably because of an impaired thrombocyte shedding. In most patients, substantial regeneration of megakaryo- and thrombopoiesis started between Day 20 and Day 30. Hematopoietic reconstitution was accompanied by a raise in the platelet values (Table 2), which, however, may be influenced to a certain degree by transfusion therapy. After platelet (and granulocyte) transfusion dependency, approximately 2 to 4 months later (End Points 3 and 4), thrombocyte counts were at the lower normal range in most patients and were accompanied by a significantly correlated number of megakaryocytes (Fig 3). In opposition to atypical micromegakaryocytes (Figs. 1a, 2a) characterizing the pretransplant bone marrow samples in patients with CML (Table 3) as well as the dysplastic megakaryocytes (Fig. 1b) immediately after BMT (End Point 2), postgraft histology revealed an enlargement of these cells with

FIGURE 2. (a-c) Megakaryocytes and (d-g) fibers before (a-d) and after (e-g) bone marrow transplantation (BMT) in patients with chronic myeloid leukemia (CML). Hypolobulated and immature megakaryocytes (a), including small precursors (b, c), are frequently found in the CML bone marrow. In some patients, there is a manifest reticulin fibrosis before BMT (d). Myelofibrosis may recur in these patients after about 2 to 3 months, generally accompanied by an unsuccessful (e) or delayed (f) hematopoietic reconstitution opposed to the majority, with undisturbed engraftment (g) showing normal-size nondysplastic megakaryocytes (arrows; $a-c 1270 \times ; d-g$ 370×). 


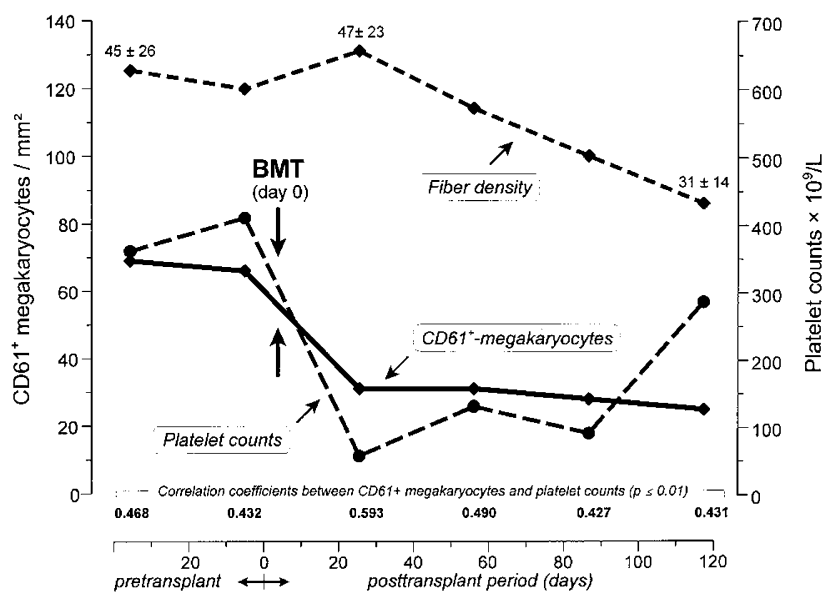

FIGURE 3. Dynamics of $\mathrm{CD} 61^{+}$megakaryocyte quantity, fiber density $\left(i \times 10^{2} / \mathrm{mm}^{2}\right)$ and platelet count during the pretransplant and posttransplant period in 127 patients with chronic myeloid leukemia and bone marrow transplantation (BMT), including correlation coefficients.

an almost normal appearance (Fig. 1c). A prevalence of normal-size megakaryocytes was observable, together with the onset of erythropoietic regeneration in the post-transplant period after successful hematopoietic recovery (Fig. 1d).

In comparison with normal bone marrow features, postgraft quantity of immature megakaryocyte precursors (Fig. 2a-c) slightly increased, which indicated a left-shifted (regenerating) megakaryopoiesis (Table 2). Planimetric measurements at these end points were in keeping with the finding that in the majority of patients, a normalization of megakaryocyte sizes occurred (Table 3). On the other hand, reappearance of atypical micromegakaryocytes in the prolonged post-transplant period was a definitive sign of leukemic relapse and was in line with the recurrence of a $\mathrm{Ph}^{1+}$ cell population (Fig. 1g). This feature is shown in a patient with an increasing number of $\mathrm{Ph}^{1+}$ metaphases in sequential biopsies less than 2 years after BMT (Fig. 4).

Before BMT (End Point 1), fiber density was not only significantly correlated $(r=0.351, P \leq .01)$ with the total number of $\mathrm{CD}^{+} 1^{+}$-megakaryocytes (Fig. 3), but also with the fraction of atypical micromegakaryocytes and precursor cells $(r=0.388, P \leq$ $.01)$. The latter were consistent with pro- and megakaryoblasts (Fig. 2b, 2c). In 34 patients with pretransplant myelofibrosis corresponding with an at least threefold increase in the normal fiber density (Fig. 2d), an insidious reappearance of reticulin fibrosis was noticeable in the areas of hematopoietic reconstitution after marrow-ablative therapy and BMT (Fig. 2e, 2f). However, retrieval of early to moderate myelofibrosis was not strictly limited to the cohort with pretransplant myelofibrosis, but was also developed in several patients without substantial fiber increase at onset. Contrasting with the
TABLE 2. Quantifications of Megakaryocyte and Reticulin Fiber Density (Mean \pm SD) per Total Marrow Area (per Hematopoietic Area in Parentheses and Platelet Counts during the Pre- and Post-transplant Period in Patients with Allogeneic Bone Marrow Transplantation (BMT) in Chronic Myeloid Leukemia

\begin{tabular}{|c|c|c|c|c|}
\hline \multirow{2}{*}{$\begin{array}{c}\text { End } \\
\text { Points* }\end{array}$} & \multicolumn{2}{|c|}{$\begin{array}{c}\text { Megakaryocyte } \\
\text { Frequency (per } \mathrm{mm}^{2} \text { ) }\end{array}$} & \multirow{2}{*}{$\begin{array}{l}\text { Fiber Density } \\
\left(i \times 10^{2} / \mathrm{mm}^{2}\right)\end{array}$} & \multirow{2}{*}{$\begin{array}{l}\text { Thrombocyte } \\
\text { Count }\left(\times 10^{9} / \mathrm{L}\right)\end{array}$} \\
\hline & $\mathrm{CD} 1^{+}$ & Precursors & & \\
\hline 0 & $\begin{array}{c}65 \pm 59 \\
(69 \pm 58)\end{array}$ & $\begin{array}{c}19 \pm 16 \\
(21 \pm 16)\end{array}$ & $\begin{array}{c}37 \pm 25 \\
(45 \pm 26)\end{array}$ & $362 \pm 270$ \\
\hline 1 & $\begin{array}{c}62 \pm 50 \\
(66 \pm 49)\end{array}$ & $\begin{array}{c}19 \pm 16 \\
(20 \pm 15)\end{array}$ & $\begin{array}{c}37 \pm 20 \\
(43 \pm 20)\end{array}$ & $411 \pm 316$ \\
\hline 2 & $\begin{array}{c}23 \pm 12 \\
(31 \pm 18)\end{array}$ & $\begin{array}{c}7 \pm 4 \\
(8 \pm 5)\end{array}$ & $\begin{array}{c}29 \pm 19 \\
(47 \pm 23)\end{array}$ & $59 \pm 55$ \\
\hline 3 & $\begin{array}{c}23 \pm 10 \\
(31 \pm 8)\end{array}$ & $\begin{array}{c}8 \pm 4 \\
(11 \pm 5)\end{array}$ & $\begin{array}{c}26 \pm 12 \\
(41 \pm 15)\end{array}$ & $121 \pm 73$ \\
\hline 4 & $\begin{array}{c}20 \pm 14 \\
(28 \pm 19)\end{array}$ & $\begin{array}{c}7 \pm 5 \\
(9 \pm 6)\end{array}$ & $\begin{array}{c}21 \pm 13 \\
(36 \pm 16)\end{array}$ & $92 \pm 80$ \\
\hline 5 & $\begin{array}{c}19 \pm 13 \\
(25 \pm 19)\end{array}$ & $\begin{array}{c}6 \pm 5 \\
(8 \pm 7)\end{array}$ & $\begin{array}{c}15 \pm 9 \\
(31 \pm 14)\end{array}$ & $287 \pm 167$ \\
\hline
\end{tabular}

* For a definition of end points, see Table 1.

Normal values: CD61 ${ }^{+}$-megakaryocytes $25 \pm 5(10,24)$, precursors $4.7 \pm 0.9$ (19), fibers $16 \pm 5(21)$, thrombocytes $150-350 \times 10^{9} / \mathrm{L}$.

undisturbed regeneration of hematopoiesis, including normal-size, nondysplastic megakaryocytes (Fig. 2g), this recurrence appeared mostly within the 2nd and 3rd month of bone marrow examination after BMT. Recurrence of patchy myelofibrosis involved 9 patients at End Point 2 and 14 patients at End Point 3 (Table 1). In these cases, reappearance of a relevant amount of reticulin fibers in the areas of recovering hematopoiesis (Fig. 2f) was significantly associated $(r=0.325, P \leq .01)$ with the quantity of megakaryocytes and as well as the amount of megakaryocyte precursors.

Regarding engraftment parameters, pretransplant (End Point 1; Table 1) fiber density exerted a distinctive impact on hematopoietic reconstitution. After BMT at End Point 3 (38 \pm 4d; Table 1), 11 of 41 patients showed delayed engraftment according to standard criteria. In keeping with features previously mentioned, this cohort was characterized by a significant increase in reticulin fibers and a tendency for a higher megakaryocyte count compared with the group with successful hematopoietic regeneration. Further, patients with disturbed engraftment and leukemic relapse had significantly higher $\mathrm{CD}^{+} 1^{+}$ megakaryocytes counts (Table 4). Similar findings were also found for the pretransplant (End Point 1; Table 1) platelet count. Here, a significant $(P \leq .03)$ difference of values $(384 \pm 306$ versus $486 \pm 336 \times$ $10^{9} / \mathrm{L}$ ) could be determined between successful as opposed to slowed engraftment.

\section{DISCUSSION}

Although extensive experience has established the widespread use of allogeneic BMT as the only curative treatment option for CML $(1,3,5,20)$, little 


\begin{tabular}{|c|c|c|c|c|c|c|}
\hline & \multicolumn{5}{|c|}{ CML } & \multirow{2}{*}{ Controls } \\
\hline & Pretreatment Biopsy & Last Before BMT & & After BMT & & \\
\hline End points & 0 & 1 & 2 & 3 & 5 & - \\
\hline Size total $\left(\mu \mathrm{m}^{2}\right)$ & $195 \pm 32(143-265)$ & $214 \pm 38(130-271)$ & $323 \pm 110(215-480)$ & $303 \pm 70(190-369)$ & $283 \pm 93(178-542)$ & $266 \pm 123$ \\
\hline $\begin{array}{l}\text { Incidence of } \\
\text { normal-sized } \\
\text { megakaryocytes } \\
\left(\geq 250 \mu \mathrm{m}^{2}\right)\end{array}$ & $24.5 \%$ & $30.3 \%$ & $54.6 \%$ & $58.7 \%$ & $74.1 \%$ & $70.0 \%$ \\
\hline $\begin{array}{l}\text { Micromegakaryocytes } \\
\text { and precursors } \\
\left(\leq 200 \mu \mathrm{m}^{2}\right.\end{array}$ & $58.0 \%$ & $51.2 \%$ & $29.0 \%$ & $25.0 \%$ & $15.5 \%$ & $17.0 \%$ \\
\hline $\begin{array}{l}\text { Ratio of nucleus/ } \\
\text { cytoplasm }\end{array}$ & $0.32 \pm 0.03$ & $0.30 \pm 0.05$ & $0.38 \pm 0.09$ & $0.32 \pm 0.08$ & $0.31 \pm 0.05$ & $0.29 \pm 0.02$ \\
\hline
\end{tabular}

BMT, bone marrow transplantation; CML, chronic myeloid leukemia.

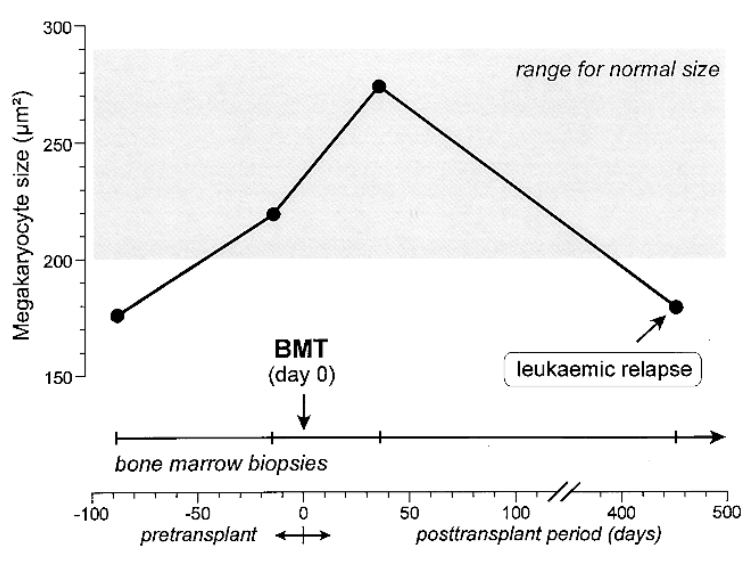

FIGURE 4. Dynamics of megakaryocyte size and outcome of allogeneic BMT in a patient with chronic myeloid leukemia and repeatedly performed trephine biopsies. BMT, bone marrow transplantation.

TABLE 4. Outcome of Allogeneic BMT in 127 Patients According to Quantity of $\mathrm{CD61}^{+}$-Megakaryocytes

\begin{tabular}{lrcc}
\hline BMT Parameter & $n$ & $\begin{array}{c}\text { Megakaryocyte Count } \\
\text { (Mean } \pm \text { SD per mm }{ }^{2} \text { ) } \\
\text { Before BMT }\end{array}$ & $\begin{array}{c}\text { Level of } \\
\text { Significance } \\
\text { P-value }\end{array}$ \\
\hline $\begin{array}{l}\text { Engraftment } \\
\quad \text { Delayed or failure }\end{array}$ & 32 & $78 \pm 69$ & \\
$\quad$ Successful & 95 & $63 \pm 44$ & .042 \\
$\quad$ Transplant failure & & & \\
$\quad$ Yes & 4 & $105 \pm 96$ & .073 \\
$\quad$ No & 123 & $65 \pm 49$ & .025 \\
Leukemic relapse & & $85 \pm 54$ & \\
$\quad$ Yes & 19 & $63 \pm 50$ & \\
No & 108 & & \\
\hline
\end{tabular}

BMT, bone marrow transplantation; CML, chronic myeloid leukemia.

information exists about the evolution of morphologic features (21-23), which reflects corresponding clinical data in the post-transplant period (1-7). Because the present study was focused on megakaryopoiesis and reticulin fibers, their mutual involvement in the generation of the myelofibrotic stroma compartment has to be regarded. In the last decade, several relevant in vitro studies have substantially increased our knowledge about the complexity and diversity of the functional interactions between abnormal megakaryocytes and the pathogenesis of myelofibrosis (13). These observations have led to the generally accepted notion that an inappropriate intramedullary release of certain growth factors (platelet-derived growth factor and transforming growth factor $\beta$ ) stimulates proliferation of nonclonally transformed fibroblasts and mediates an enhanced deposition of interstitial and basement membrane collagen $(14,15)$. These close biological relationships were able to explain the frequently detectable histotopographical proximity between atypical megakaryocytes and the ensuing network of reticulin fibers $(8-12,24)$.

The current hypothesis for the pathogenesis of myelofibrosis was further supported by findings in patients with acute micromegakaryocytic leukemia. Significant correlations could be determined between in vitro growth factor release, bone marrow stroma turnover, and mass of atypical micromegakaryocytes $(16,17)$. In line with these results, significant associations between the number of $\mathrm{CD}^{+} 1^{+}$megakaryocytes and their precursor fraction with the content of argyrophilic (reticulin-collagen) fibers were calculable by morphometric quantification (24). The present study extends these findings in that our data indicate that in the post-transplant period, regeneration of megakaryopoiesis is not only linked with a reduction of transfusiondependent thrombocytopenia, but may be also associated with an insidiously occurring retrieval of reticulin fibrosis in the engrafted marrow. This feature was predominantly expressed in those patients with CML with a manifest myelofibrosis before BMT, but occasionally it may develop also in the pregraft nonfibrotic bone marrow. The assumption that normal (i.e., nonclonally transformed) megakaryocytes of the donors are capable of growth factor release and fibroblast stimulation is in keeping with corresponding in vitro findings on isolated and enriched megakaryocytes derived from healthy donors (25-27). 
In this context, it is tempting to speculate that the pretransplant conditioning regimens not only generated space or microenvironmental niches for the regeneration of the hematopoietic stem cells of the donor, but also could have failed to ablate totally all cell populations of the recipient. The latter may in particular apply to the more resistant cells, such as fibroblasts or fibrocytes (28). Experimental data are in line with the finding that depending on the mode of marrow-ablative treatment (29), the microenvironment remains qualitatively altered several months after BMT. This implies a decreased capacity to support regenerating hematopoiesis and cytokine secretion (30). Moreover, bone marrow fibroblasts of patients with CML revealed a tendency for an increase growth and collagen synthesis (31); however, they display no clonal evolution (32-34). For this reason, it is presumed that stimulated nonclonal fibroblasts of the recipient patient involved in pregraft myelofibrosis may have survived myeloablative therapy. Accordingly, they are able to exert a corresponding effect on the cytokine-mediated development of post-transplant fibrosis in the donor marrow. Taken together, the data presented here support the postulated functional relationships between megakaryopoiesis, cytokine, and growth factor release involved in the synthesis of myelofibrotic extracellular matrix proteins (13-15).

Compelling evidence has been produced that in CML, megakaryopoiesis is characterized by the predominance of atypical microformes showing a dwarf-like appearance with dense hypolobulated nuclei $(8-11,19,24)$. In addition, it has been pointed out that megakaryocyte size, nuclear size, degree of nuclear lobulation, and ploidy were significantly correlated with each other (35). After a number of investigations involving different techniques such as flow cytometric analysis, determination of DNA content, and size and ploidy measurements, it has been demonstrated convincingly that opposed to the normal state, in CML, the majority of megakaryocytes are not only very small but also hypoploid $(36,35)$. For this reason, presence of micromegakaryocytes in patients presenting with thrombocytosis is a diagnostic hallmark of CML: they distinguish this condition from the other eventually thrombocythemic subtypes of chronic myeloproliferative disorders (37-39).

Furthermore, fluorescence in situ hybridization reveals that a normalization of megakaryocyte size after interferon- $\alpha$ therapy in CML is significantly associated with a loss of the bcr/abl translocation sites. Therefore, this feature indicates a (partial) recovery of normal hematopoiesis (40). Consequently, the increased number of normal-size megakaryocytes in the post-transplant period (Table 3) implies an absence of a leukemic cell population, which is in accordance with corresponding cytogenetic and molecular-biological findings in these patients. In support of this fact, reappearance of abnormal micromegakaryocytes was compatible with the clinical finding of a leukemic relapse (Fig. 1e). This feature emphasizes the impact of a scrutinized histologiccytologic assessment of megakaryopoiesis in the posttransplant period for the early detection of an eventually occurring expansion of the leukemic cell lineage.

A significant correlation between platelet count and quantity of megakaryocytes was determinable in the CML bone marrow before BMT and after completed hematopoietic reconstitution (Fig. 3). Although in the early post-transplant period (End Point 2; Table 1) the number of megakaryocytes reached the lower limit of the normal value (10), there was a transfusion-dependent thrombocytopenia (Table 2). It is well known that after myeloablative therapy, significant damage of the bone marrow stromal compartment occurs that greatly hampers the normal function of the microenvironment (30). Corresponding lesions are demonstrable by a conspicuous scleredema and abnormal cytology of regenerating hematopoiesis (23). The dysplastic features that in particular are observable in megakaryocytes (i.e., a striking increase in the nuclear-cytoplasmic ratio) may account for a functional impairment of platelet shedding $(22,23)$ and are grossly comparable with situations encountered in myelodysplastic syndromes. Regeneration of regular-size megakaryocytes with nondisturbed cytology resembling the normal state (10) is evident only after Day 20 after BMT (Table 3).

Finally, discussion and controversy arises when addressing the relevance of bone marrow fibrosis for hematopoietic engraftment after marrowablative therapy and BMT. There seems to be general agreement that myelo-ablative regimens are causing a significant reversal of myelofibrosis (21$23,47)$. In the beginning, this fibrolytic effect is also observable in the bone marrow specimens of patients who present with manifest reticulin fibrosis before BMT. On the other hand, a retrieval of reticulin fibrosis accompanied by an increased number of megakaryocytes was observed in the areas of regenerating hematopoiesis several months after BMT (Fig. 2e, 2f) and predominantly in those patients with unsuccessful engraftment. A conflict of opinion has been repeatedly expressed concerning the influence of myelofibrosis on engraftment parameters. To date, relevant studies included series of patients with myelofibrosis accompanying a heterogeneous variety of underlying conditions. These ranged from agnogenic myeloid metaplasia to myelodysplastic syndromes and malignant lymphomas $(42,43)$.

In contrast to the previously published data that severe myelofibrosis was responsible for a signifi- 
cantly slower rate of myeloid recovery and a longer dependence on platelet and red blood cell transfusion (42), this result was not supported by a consecutively conducted study from the same group (43). According to our findings, which are exclusively based on patients with CML and which involved morphometric quantification with standardized end points during the post-transplant period, a relevant myelofibrosis impairs successful bone marrow engraftment. In keeping with the significant functional relationship between megakaryocytes and generation of fibers (13-17), an adverse outcome (delayed engraftment and leukemic relapse) was associated with an increased number of megakaryocytes before BMT and a correspondingly higher pretransplant platelet value with a delayed engraftment.

In conclusion, histologic and morphometric evaluations on repeatedly performed bone marrow trephine biopsies in patients before and after BMT for CML at standardized end points provide persuasive evidence for a close relationship between megakaryocyte number and size and the outcome of engraftment and also between megakaryopoiesis, including precursors and generation of (reticulin) fibrosis.

Acknowledgments: The authors thank Mrs. B. Rosenbach, Mrs. M. Wonschick, and Mrs. H. Tilemann for their excellent technical assistance.

\section{REFERENCES}

1. Appelbaum FR, Clift R, Radich J, Anasetti C, Buckner CD. Bone marrow transplantation for chronic myelogenous leukemia. Semin Oncol 1995;22:405-41.

2. Beelen DW, Graeven U, Elmaagacli AH, Niederle N, Kloke O, Opalka B, et al. Prolonged administration of interferon- $\alpha$ in patients with chronic-phase Philadelphia chromosomepositive chronic myelogenous leukemia before allogeneic bone marrow transplantation may adversely affect transplant outcome. Blood 1995;36:2981-90.

3. Clift RA, Anasetti C. Allografting for chronic myeloid leukaemia. Baillieres Clin Haematol 1997;10:319-36.

4. Lamparelli T, Van Lint MT, Gualandi F, Occhini D, Barbanti M, Sacchi N, et al. Bone marrow transplantation for chronic myeloid leukemia (CML) from unrelated and sibling donors: single center experience. Bone Marrow Transplant 1997;20: 1057-62.

5. Van Rhee F, Szydlo RM, Hermans J, Devergy A, Frassoni F, Arcese W, et al., for the Chronic Leukemia Working Party of the European Group for Blood and Marrow Transplantation. Long-term results after allogeneic bone marrow transplantation for chronic myelogenous leukemia in chronic phase: a report from the Chronic Leukemia Working Party of the European Group for Blood and Marrow Transplantation. Bone Marrow Transplant 1997;20:553-60.

6. Dini G, Lamparelli T, Rondelli R, Lanino E, Barbanti M, Costa C, et al. Unrelated donor marrow transplantation for chronic myelogenous leukaemia. Br J Haematol 1998;102:544-52.

7. Hansen JA, Gooley TA, Martin PJ, Appelbaum F, Chauncey TR, Clift AR, et al. Bone marrow transplants from unrelated donors for patients with chronic myeloid leukemia. N Engl J Med 1998;338:962-8.

8. Burkhardt R, Bartl R, Jaeger K, Frisch B, Kettner G, Mahl G, et al. Chronic myeloproliferative disorders (CMPD). Pathol Res Pract 1984;179:131-86.

9. Georgii A, Vykoupil KF, Buhr T, Choritz H, Doehler U, Kaloutsi V, et al. Chronic myeloproliferative disorders in bone marrow biopsies. Pathol Res Pract 1990;186:3-27.

10. Thiele J, Fischer R. Megakaryocytopoiesis in haematological disorders: diagnostic features of bone marrow biopsies. An overview. Virchows Archiv A Pathol Anat 1991;418:87-97.

11. Buhr T, Choritz H, Georgii A. The impact of megakaryocyte proliferation for the evolution of myelofibrosis. Histological follow-up study in 186 patients with chronic myeloid leukemia. Virchows Arch A Pathol Anat 1992;420:473-8.

12. Bartl R, Frisch B, Wilmanns W. Potential of bone marrow biopsy in chronic myeloproliferative disorders (MPD). Eur J Haematol 1993;50:41-52.

13. Reilly JT. Pathogenesis of idiopathic myelofibrosis: present status and future directions. Br J Haematol 1994;88:1-8.

14. Kimura A, Katoh O, Hyodo H, Kuramoto A, Satow Y. Platelet derived growth factor expression, myelofibrosis and chronic myelogenous leukemia. Leuk Lymphoma 1995;18:237-42.

15. Martyré MC. TGF- $\beta$ and megakaryocytes in the pathogenesis of myelofibrosis in myeloproliferative disorders. Leuk Lymphoma 1995;20:39-44.

16. Terui T, Niitsu Y, Mahara K, Fujisaki Y, Urushizaki Y, Kohogo $\mathrm{Y}$, et al. The production of transforming growth factor- $\beta$ in acute megakaryoblastic leukemia and its possible implication in myelofibrosis. Blood 1990;75:1540-8.

17. Reilly JT, Barnett D, Dolan G, Forrest P, Eastham J, Smith A. Characterization of an acute micromegakaryocytic leukemia: evidence for the pathogenesis of myelofibrosis. Br J Haematol 1993;83:58-62.

18. Gatter KC, Cordell JL, Turley H, Heryet A, Kiefer N, Anstee DJ, et al. The immunohistological detection of platelet, megakaryocytes and thrombi in routinely processed specimens. Histopathology 1988;13:257-67.

19. Thiele J, Wagner S, Weuste R, Dienemann D, Wienhold S, Zankovich $\mathrm{R}$, et al. An immunomorphometric study on megakaryocyte precursor cells in bone marrow tissue from patients with chronic myeloid leukemia (CML). Eur J Haematol 1990;44:63-70.

20. Gratwohl A, Hermans J, Niederwieser D, Frassoni F, Arcese W, Gahrton G, et al. for the Chronic Leukemia Working Party of the European Group for Bone Marrow Transplantation. Bone marrow transplantation for chronic myeloid leukemia: long-term results. Bone Marrow Transplant 1993;12:509-16.

21. Van den Berg H, Kluin PM, Vossen JM. Early reconstitution of haematopoiesis after allogeneic bone marrow transplantation: a prospective histopathological study of bone marrow biopsy specimens. J Clin Pathol 1990;43:365-9.

22. Rousselet MC, Kerjean A, Guyétant S, François S, SaintAndré JP, Ifrah N. Histopathology of bone marrow after allogeneic bone marrow transplantation for chronic myeloid leukaemia. Pathol Res Pract 1996;192:790-5.

23. Hurwitz N. Bone marrow trephine biopsy changes following chemotherapy and/or bone marrow transplantation. Curr Diagn Pathol 1997;4:196-202.

24. Thiele J, Kvasnicka HM, Fischer R. Bone marrow histopathology in chronic myelogenous leukemia (CML) - evaluation of distinctive features with clinical impact. Histol Histopathol 1999;14:1241-56.

25. Wickenhauser C, Hillienhof A, Jungheim K, Lorenzen J, Ruskowski $\mathrm{H}$, Hansmann ML, et al. Detection and quantification of transforming growth factor $\beta$ (TGF- $\beta$ ) and platelet-derived growth factor (PDGF) release by normal human megakaryocytes. Leukemia 1995;9:310-5. 
26. Wickenhauser C, Lorenzen J, Thiele J, Hillienhof A, Jungheim $\mathrm{K}$, Schmitz B, et al. Secretion of cytokines (IL-1a, IL-3, and IL6 and GM-CSF) by normal human bone marrow megakaryocytes. Blood 1995;85:685-91.

27. Schmitz B, Thiele J, Witte O, Kaufmann R, Wickenhauser C, Fischer R. Influence of cytokines (IL-1a. IL-11, GM-CSF) on megakaryocyte-fibroblasts interactions in normal human bone marrow. Eur J Haematol 1995;55:24-32.

28. Martin PJ. Determinants of engraftment after allogeneic marrow transplantation. Blood 1992;79:1647-50.

29. Salomon O, Lapidot T, Terenzi A, Lubin I, Rabi I, Reisner Y. Induction of donor-type chimerism in murine recipients of bone marrow allografts by different radiation regimens currently used in treatment of leukemia patients. Blood 1990;76: 1872-8.

30. Domenech J, Roingeard F, Hérault O, Truglio D, Desbois I, Colombat $\mathrm{P}$, et al. Changes in functional capacity of marrow stromal cells after autologous bone marrow transplantation. Leuk Lymphoma 1998;29:533-46.

31. Yonekura S, Nago T, Arimori S. Increased growth and collagen synthesis of bone marrow fibroblasts from patients with chronic myelocytic leukaemia. Br J Haematol 1985;61:93-99.

32. Greenberg BR, Wilson FD, Woo L, Jenks HM. Cytogenetics of fibroblastic colonies in $\mathrm{Ph}^{1}$-positive chronic myelogenous leukemia. Blood 1978;51:1039-44.

33. O'Brien S, Kantarjian H, Shtalrid M, Blick M, Beran M, Talpaz M. Lack of cluster region rearrangement in marrow fibroblasts of patients with Philadelphia chromosome-positive chronic myelogenous leukemia. Hematol Pathol 1988;2:25-9.

34. Golde DW, Hocking WG, Quan SG, Sparkes RS, Gale RP. Origin of human bone marrow fibroblasts. Br J Haematol 1980;44:183-7.

35. Levine RF, Hazzard KC, Lamberg JD. The significance of megakaryocyte size. Blood 1982;60:1122-31.
36. Ishibashi T, Ruggeri ZM, Harker LA, Burstein SA. Separation of human megakaryocytes by state of differentiation on continuous gradients of Percoll: size and ploidy analysis of cells identified by monoclonal antibody to glycoprotein IIb/IIIa. Blood 1986;67:1286-92.

37. Tomer A, Friese P, Conklin R, Bales W, Archer L, Harker LA, et $a l$. Flow cytometric analysis of megakaryocytes from patients with abnormal platelet counts. Blood 1989;74:594-601.

38. Ridell B, Kutti J, Revesz P, Bergström S, Enerbäck L. DNA content and nuclear size of megakaryocytes in thrombocythaemia. APMIS 1990;98:845-53.

39. Woods MJ, Greaves M, Trowbridge EA. Megakaryocyte ploidy in thrombocytosis: improved microdensitometric measurement with a new image analysis system. Eur J Haematol 1990;44:220-6.

40. Thiele J, Schmitz B, Gross H, Kvasnicka HM, Niederle N, Leder LD, et al. Fluorescence in-situ hybridization (FISH) reveals that in chronic myelogenous leukaemia (CML) following interferon- $\alpha$ therapy, normalization of megakaryocyte size is associated with the loss of bcr/abl translocation. Histopathology 1997;31:215-21.

41. McGlave PB, Brunning RD, Hurd DD, Kim KH. Reversal of severe bone marrow fibrosis and osteosclerosis following allogeneic bone marrow transplantation for chronic granulolytic leukaemia. Br J Haematol 1982;52:189-94.

42. Rajantie J, Sale GE, Deeg HJ, Amos D, Appelbaum FR, Storb $\mathrm{R}$, et al. Adverse effect of severe marrow fibrosis on hematologic recovery after chemoradiotherapy and allogeneic bone marrow transplantation. Blood 1986;67:1693-7.

43. Soll E, Massumoto C, Clift RA, Buckner CD, Appelbaum FR, Storb R, et al. Relevance of marrow fibrosis in bone marrow transplantation: a retrospective analysis of engraftment. Blood 1995;86:4667-73. 